\title{
Alterations in microRNA expression profile in rabies virus-infected mouse neurons
}

\author{
N. SHI ${ }^{1}$, X. Y. ZHANG ${ }^{1}$, C.Y. DONG, J. L. HOU ${ }^{1}$, M. L. ZHANG ${ }^{1}$, Z. H. GUAN ${ }^{1}$, Z. Y. LI², M. DUAN ${ }^{*}$
}

\begin{abstract}
${ }^{1}$ Key Laboratory of Zoonosis, Ministry of Education, Institute of Zoonosis, Jilin University, 130062 Changchun, P. R. China; ${ }^{2}$ Key Laboratory of Jilin Province for Zoonosis Prevention and Control, Institute of Military Veterinary, Academy of Military Medical Sciences, 130062 Changchun, P. R. China
\end{abstract}

Received September 3, 2013; accepted April 23, 2014

\begin{abstract}
Summary. - Rabies virus (RABV) is known to cause a fatal infection in many mammalian species, yet its pathogenesis remains poorly understood. This study was performed to analyze the microRNA (miRNA) expression profiles in RABV-infected primary neurons of mice. A total of 53 miRNAs were found to be differentially expressed in RABV-infected samples compared with mock samples in a time-dependent manner. Among them, the expression of ten miRNAs was validated by real-time RT-PCR. Potential target genes of differentially expressed miRNAs were predicted by TargetScan. Further bioinformatics analysis indicated that these predicted targets were overrepresented in neuronal function-related Gene Ontology (GO) terms and biological pathways. The results of this study suggest that RABV may cause neuronal dysfunction by regulating cellular miRNA expression.
\end{abstract}

Keywords: rabies virus; microRNA; expression; microarray; neurons; mouse

\section{Introduction}

RABV, (the genus Lyssavirus, the family Rhabdoviridae) is a highly neurotropic virus that spreads along neural pathways and invades the CNS, where it causes an acute infection (Schnell et al., 2010). Although significant advances have been made in rabies prevention and control, rabies remains a major threat to public health (Hemachudha et al., 2002). Despite the catastrophic clinical outcome of rabies encephalomyelitis, the fact that inflammatory changes and degenerative neuronal changes may be minimal, or even absent, under natural conditions indicates that neuronal dysfunction, rather than neuronal death, is likely responsible for the clinical disease and fatal outcome of rabies under natural

“E-mail: duan_ming@jlu.edu.cn; phone: +86-431-87836715. Abbreviations: CNS $=$ central nervous systems; DAVID = Database for Annotation, Visualization, and Integrated Discovery; $\mathrm{FDR}=$ false discovery rate; $\mathrm{GO}=\mathrm{Gene}$ Ontology; KEGG = Kyoto Encyclopedia of Genes and Genomes; miRNA = microRNA; p.i. = post infection; $\mathrm{RABV}=$ rabies virus conditions (Jogai et al., 2000; Fu et al., 2005). Although there are several hypotheses under investigation at the present time, the fundamental basis for neuronal dysfunction in rabies has not yet been demonstrated.

miRNAs, an extensive class of non-coding RNA molecules, negatively regulate gene expression and play essential roles in many critical cellular processes such as development, differentiation, proliferation, and hematopoiesis (Ambros, 2004; Bartel, 2004). As is well known, viral infection represents a great change in the host cellular environment that generally results in dramatic changes in cellular gene expression. Similarly, because most miRNAs are also transcribed by RNA polymerase II, using many of the same cellular transcription factors, the viral infection would also affect their patterns of expression. It is expected that viruses may gain an advantage by reshaping the cellular miRNA composition to directly or indirectly affect viral replication and pathogenesis (Cullen, 2010). For example, a cellular miRNA required for virus replication is $\mathrm{miR}-122$, which is important to the complete life cycle of hepatitis C virus (Jopling et al., 2005); human T-cell lymphotropic virus type 1 modulates 
the expression of a number of cellular miRNAs in order to control T-cell differentiation (Bellon et al., 2009); human cytomegalovirus selectively manipulates the expression of miR-100 and miR-101 to facilitate its own replication (Wang et al., 2008); Epstein-Barr virus LMP1 induces miR$29 \mathrm{~b}$, which results in miR-29b-mediated downregulation of T-cell leukemia gene 1 , a protein with roles in cell survival and proliferation (Anastasiadou et al., 2010); miR-29c is involved in A549 cell apoptosis induced by influenza virus infection through repression of antiapoptotic factor BCL2L2 (Guan et al., 2012); CCL8/MCP-2 is a target for miR-146a in HIV-1-infected microglia, as overexpression of miR-146a prevents HIV-induced secretion of MCP-2 chemokine (Rom et al., 2010).

Given the above mentioned information, this study was aimed to identify potential dysregulated miRNAs in RABVinfected neurons. We carried out a miRNA expression profile of primary neurons of mice infected with mock and RABV, and performed real-time RT-PCR validation of several differentially expressed miRNAs. Functional enrichment of the predicted miRNA target genes presented neuronal functionrelated GO terms and intracellular signaling pathways, which would be very helpful in understanding neuronal dysfunction in rabies.

\section{Materials and Methods}

Virus, cells and animals. The MRV strain C (GenBank Acc. No. DQ875050.1) of street RABV, a canine RABV variant isolated from the Henan province of China, was grown in mouse N2a neuroblastoma cells. Primocultures of neurons were derived from the cortex of embryonic day 16.5 (E 16.5) C57BL/6 J mice. The cortexes were incubated with $0.25 \%$ trypsin, and $50 \mu \mathrm{l}$ of $10 \mathrm{mg} / \mathrm{ml}$ DNase I stock was added for $30 \mathrm{sec}$ to break down the DNA and avoid tissue clumping. The dissociated neurons were initially plated in DMEM with $10 \%$ FBS (GIBCO) at the plating density of $1 \times 10^{5}$ cells per $\mathrm{cm}^{2}$. After $2 \mathrm{hr}$, the plating medium was carefully replaced with equilibrated neurobasal media containing B27 supplement (GIBCO) and $2 \mathrm{mmol} / \mathrm{l} \mathrm{L}$-glutamine (Invitrogen). The cells grew for up to 1 week without any further change of medium. The cells were infected with RABV at $10 \mathrm{TCID}_{50}$ per well in 6 well-plates. C57BL/6J mice had access to food and water ad libitum and were challenged with RABV by intracranial inoculation at a dose of 10 $\mathrm{LD}_{50}$ per $30 \mu \mathrm{l}$. All experiments with live virus challenge were carried out in Biosafety Level 2 facilities.

miRNA microarray. Total RNA was extracted from primocultures of neurons infected with RABV at $0,48,96$, and 144 hr post infection (p.i.) using TRIZOL reagent (Invitrogen). The RNA samples were submitted to LC Sciences (Houston, TX) for quality control, processing and miRNA expression analysis in accordance with their specifications. All samples met quality control standards. Samples were hybridized to individual mouse
miRNA microarray (LC Sciences), which were current with Sanger miRBase version 16.0. Data were analyzed by first subtracting the background and then normalizing the signal using a LOWESS method.

Real-time RT-PCR. For analysis of mature miRNAs, TaqMan miRNA assays (Applied Biosystems) were used. Total RNA was reverse transcribed using a miRNA-specific primer and Multiscribe reverse transcriptase. Real-time PCR was performed with a miRNA-specific probe according to the manufacturer's instructions. All reactions were run in triplicate on an Applied Biosystems 7500 Real-Time PCR System. Expression of the miRNA of interest was normalized to snRNA RNU6B and relative expression was calculated using the $2^{-\Delta \Delta \mathrm{Ct}}$ method. Data were expressed as mean \pm standard deviation (SD) from at least three separate experiments.

Prediction of target genes of miRNAs. TargetScan (http://www. targetscan.org/) is a web resource that predicts biological targets of miRNAs by searching for the presence of $8 \mathrm{mer}$ and $7 \mathrm{mer}$ sites that match the seed region of each miRNA (Lewis et al., 2005). We used TargetScan (Release 6.2) to predict target genes of miRNAs.

GO enrichment and biological pathway analyses of predicted target genes. All of the predicted genes were annotated with their GO information. Biological pathways were defined by Kyoto Encyclopedia of Genes and Genomes (KEGG). The GO enrichment and biological pathway analysis were performed using the webbased tool, Database for Annotation, Visualization, and Integrated Discovery (DAVID, http://david.abcc.ncifcrf.gov/) (Huang da et al., 2009). This software uses enrichment or overrepresentation analysis to determine if gene sets are statistically overrepresented in the identified gene clusters or biological pathways more than a random chance as determined by the modified Fisher's exact $P$-value. $P$-values were adjusted using the method of Benjamini and Hochberg to control the false discovery rate (FDR) (Hochberg et al., 1990).

\section{Results}

\section{Effect of RABV infection on miRNA profile in cultured} mouse neurons

To get an overview of host neuronal miRNA expression in response to $\mathrm{RABV}$ infection, we analyzed primocultures of neurons infected with RABV at $0,48,96$, and $144 \mathrm{hr}$ p.i. miRNAs whose relative expression levels showed a fold change $\geq 2$ and $P \leq 0.01$ were considered significantly upregulated, and those with fold change $\leq-2$ and $P \leq 0.01$ were considered significantly down-regulated. A total of 53 miRNAs were found to be differentially expressed in a time-dependent manner after infection. As shown in Fig. 1a, two distinct trends were presented, in which one trend was continuously upregulated with 19 miRNAs, while the other was continuously downregulated with 34 miRNAs. 
(a)

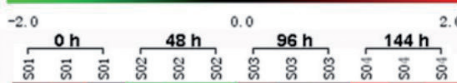

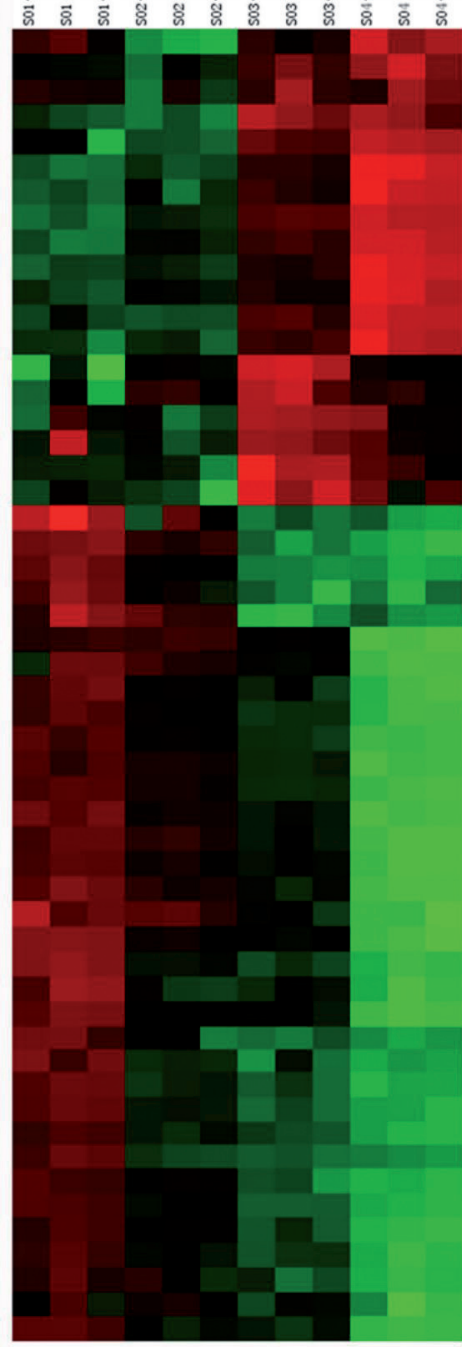

$m m u-m i R-466 i-5 p$ mum-let-7e mmu-let-7f mmu-miR-29a $m u-m i R-21$ mmu-miR-15a $m m u-m i R-466 m-3 p$ mmu-miR-1934 mmu-miR -3110 * mutur (1) mu-miR- $574-5 p$ $m m u-m i R-15 a *$ mmu-miR-705 mmu-miR-193b* mmu-miR-153 $m m u-m i R-680$ mmu-miR-1966 mmu-miR-1897-5p $m m u-m i R-466 h-3 p$ mu $-30 \mathrm{R}-3 \mathrm{p}$ mmu $-m i R-125 a-5 p$ mmu-miR-351 mmu-miR-667 $m m u-m i R-667$
$m m u-m i R-9 *$ $m m u-m i R-9 *$
$m m u-m i R-137$ $m m u-m i R-9$ mu-miR-151-3p mmu-miR-1983 mmu-miR-20a mmu-miR-128 mmu-miR-25 mmu-miR-25 mmu-miR-132 $m m u-m i R-23 a$ mmu-miR-23b $m m u-m i R-92 a$
$m m u-m i R-208 a-5$ mmu-miR-208a-
mmu-miR-92b* $m m u-m i R-181 a$ mmu-miR-26a mmu-miR-151-5p mmu-miR-720 mu-miR-130 mmu-miR-103 mum-miR-10 mmu $-m i R-124$ min-18 (18a mulu mu-mik-106 mmu-miR-30d $m m u-m i R-344$ mmu-miR-30c mu-miR-99b
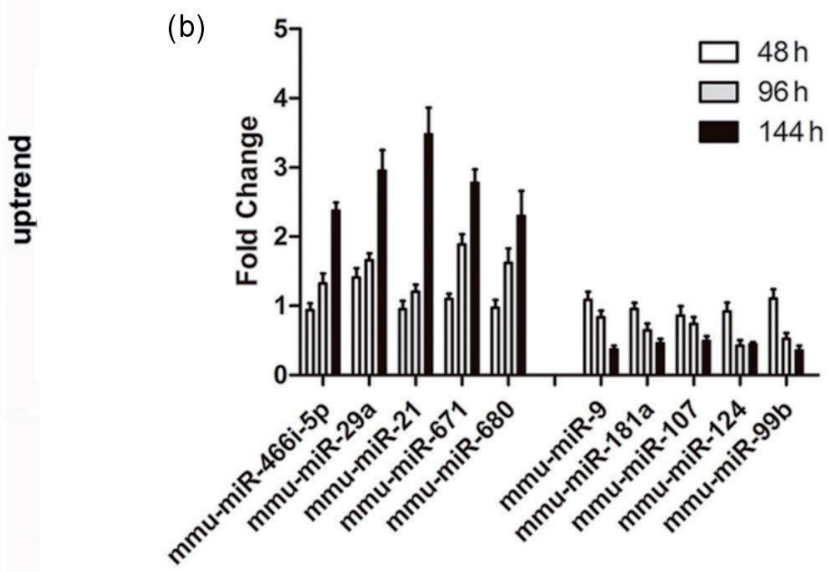

(c)
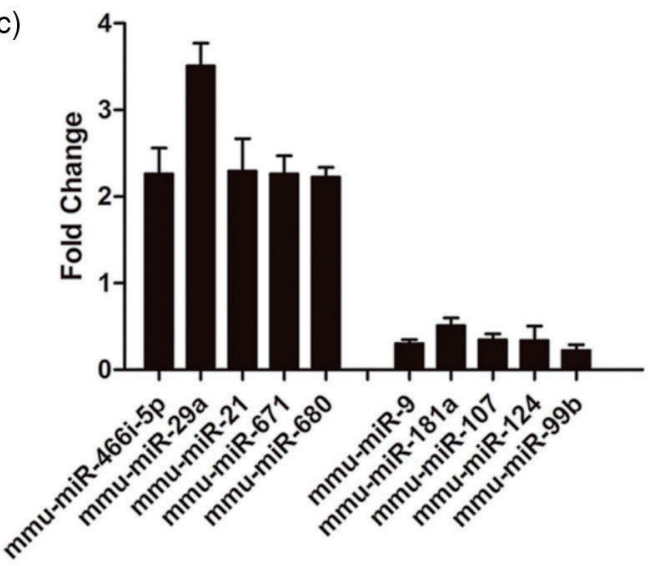

Fig. 1

Effect of RABV infection on miRNA profile in mouse neurons

(a) Two-way hierarchical cluster map of significantly up- or downregulated miRNAs in cultured neurons. (b) Selected differentially expressed miRNAs in cultured neurons as assayed by real-time RT-PCR. (c) Selected differentially expressed miRNAs in hippocampal neurons as assayed by real-time RT-PCR. Bars are represented from three independent experiments.

To verify the data from microarray analysis, we used realtime RT-PCR to detect 10 selected differentially expressed miRNAs, including miR-466i-5p, miR-29a, miR-21, miR-671, miR-680, miR-let-7e, miR-15a, miR-574-5p, miR-705, miR153, miR-125a-5p, miR-107, miR-124, miR-181a, miR-9, miR-99b, miR-20a, miR-23b, miR-720, on RNA samples from primocultures of neurons (Fig. 1b). Furthermore, these selected miRNAs were also validated on hippocampi of mice challenged with RABV at moribund stage compared to mock infection (Fig. 1c). Although differences were observed between neuronal culture and hippocampal tissue, the results showed the same expression patterns of differentially expressed miRNAs. These results demonstrate that host neuronal miRNAs could be dysregulated by RABV infection.

Target prediction and GO enrichment analysis of target genes of differentially expressed miRNAs

To predict possible target genes for differentially expressed miRNAs, we searched the TargetScan mouse database. TargetScan successfully predicted target genes of 48 differentially expressed miRNAs. A total of 6,172 
Table 1. Significant neuronal function-related GO annotations for the upregulated miRNAs

\begin{tabular}{|c|c|c|c|c|}
\hline GO term & Count $^{\mathrm{a}}$ & $\%^{\mathrm{b}}$ & $P$-value ${ }^{c}$ & $\mathrm{FDR}^{\mathrm{d}}$ \\
\hline \multicolumn{5}{|l|}{ Biological process } \\
\hline GO:0035295 tube development & 116 & 2.993548 & $1.77 \mathrm{E}-17$ & $3.32 \mathrm{E}-14$ \\
\hline GO:0030182 neuron differentiation & 156 & 4.025806 & $2.15 \mathrm{E}-17$ & 4.02E-14 \\
\hline GO:0031175 neuron projection development & 90 & 2.322581 & $8.83 \mathrm{E}-12$ & $1.65 \mathrm{E}-08$ \\
\hline GO:0007169 transmembrane receptor protein tyrosine kinase signaling pathway & 80 & 2.064516 & $8.25 \mathrm{E}-11$ & $1.54 \mathrm{E}-07$ \\
\hline GO:0048812 neuron projection morphogenesis & 75 & 1.935484 & $9.78 \mathrm{E}-11$ & $1.83 \mathrm{E}-07$ \\
\hline GO:0007264 small GTPase mediated signal transduction & 99 & 2.554839 & $1.10 \mathrm{E}-10$ & $2.05 \mathrm{E}-07$ \\
\hline GO:0007409 axonogenesis & 69 & 1.780645 & $8.35 \mathrm{E}-10$ & $1.56 \mathrm{E}-06$ \\
\hline GO:0016192 vesicle-mediated transport & 152 & 3.922581 & $1.40 \mathrm{E}-09$ & $2.63 \mathrm{E}-06$ \\
\hline GO:0046903 secretion & 83 & 2.141935 & 1.27E-08 & $2.38 \mathrm{E}-05$ \\
\hline GO:0030001 metal ion transport & 142 & 3.664516 & $1.51 \mathrm{E}-08$ & 2.82E-05 \\
\hline GO:0007268 synaptic transmission & 69 & 1.780645 & 5.92E-08 & $1.11 \mathrm{E}-04$ \\
\hline GO:0007411 axon guidance & 45 & 1.16129 & $6.03 \mathrm{E}-08$ & $1.13 \mathrm{E}-04$ \\
\hline GO:0046907 intracellular transport & 135 & 3.483871 & $1.86 \mathrm{E}-07$ & $3.47 \mathrm{E}-04$ \\
\hline GO:0010941 regulation of cell death & 168 & 4.335484 & 2.02E-07 & $3.78 \mathrm{E}-04$ \\
\hline GO:0050808 synapse organization & 27 & 0.696774 & $5.81 \mathrm{E}-07$ & 0.001085 \\
\hline GO:0006836 neurotransmitter transport & 37 & 0.954839 & $8.80 \mathrm{E}-07$ & 0.001644 \\
\hline GO:0050767 regulation of neurogenesis & 51 & 1.316129 & $4.28 \mathrm{E}-06$ & 0.007995 \\
\hline GO:0007269 neurotransmitter secretion & 21 & 0.541935 & 4.61E-06 & 0.008617 \\
\hline GO:0030029 actin filament-based process & 62 & 1.6 & $1.15 \mathrm{E}-05$ & 0.02152 \\
\hline GO:0001764 neuron migration & 31 & 0.8 & $2.29 \mathrm{E}-05$ & 0.042745 \\
\hline \multicolumn{5}{|l|}{ Cellular component } \\
\hline GO:0045202 synapse & 133 & 3.432258 & $2.77 \mathrm{E}-20$ & $4.09 \mathrm{E}-17$ \\
\hline GO:0043005 neuron projection & 104 & 2.683871 & $1.09 \mathrm{E}-16$ & $1.67 \mathrm{E}-13$ \\
\hline GO:0030425 dendrite & 52 & 1.341935 & $1.05 \mathrm{E}-10$ & $1.55 \mathrm{E}-07$ \\
\hline GO:0030424 axon & 46 & 1.187097 & $5.33 \mathrm{E}-08$ & 7.87E-05 \\
\hline GO:0031410 cytoplasmic vesicle & 148 & 3.819355 & 6.97E-08 & $1.03 \mathrm{E}-04$ \\
\hline GO:0034703 cation channel complex & 42 & 1.083871 & $8.70 \mathrm{E}-08$ & $1.29 \mathrm{E}-04$ \\
\hline GO:0031982 vesicle & 150 & 3.870968 & $9.77 \mathrm{E}-08$ & $1.44 \mathrm{E}-04$ \\
\hline GO:0005856 cytoskeleton & 283 & 7.303226 & $3.68 \mathrm{E}-07$ & $5.44 \mathrm{E}-04$ \\
\hline GO:0045211 postsynaptic membrane & 48 & 1.23871 & $1.84 \mathrm{E}-06$ & 0.002715 \\
\hline GO:0005912 adherens junction & 42 & 1.083871 & $2.84 \mathrm{E}-06$ & 0.004196 \\
\hline GO:0034702 ion channel complex & 56 & 1.445161 & $3.15 \mathrm{E}-06$ & 0.004646 \\
\hline GO:0019717 synaptosome & 29 & 0.748387 & $1.40 \mathrm{E}-05$ & 0.020692 \\
\hline GO:0014069 postsynaptic density & 24 & 0.619355 & $2.48 \mathrm{E}-05$ & 0.036536 \\
\hline GO:0015630 microtubule cytoskeleton & 123 & 3.174194 & $2.90 \mathrm{E}-05$ & 0.042794 \\
\hline
\end{tabular}

${ }^{a}$ Numbers of target genes involved in the specific GO term. ${ }^{b}$ Percentages represent involved genes/total genes annotated with GO information. ${ }^{\mathrm{c}}$ Enrichment $P$-value with modified Fisher's exact test. ${ }^{\mathrm{d}} \mathrm{FDR}$ stands for the false discovery rate using Benjamini \& Hochberg procedure.

genes were predicted as target genes for up-regulated miRNAs, while 14,780 genes were predicted for downregulated miRNAs. To better understand the potential role of miRNA in the pathogenesis of RABV, we submitted the predicted target gene lists to DAVID database for performing functional annotation. As shown in table 1 and 2, the neuronal function-related GO biological processes and cellular components were significantly over-represented $(P<0.01$ and FDR $<0.05)$, respectively. Furthermore, the target genes were also enriched into biological pathways related to neuronal function $(P<0.01$ and FDR $<0.05)$
(Table 3). These results suggest that miRNAs with significant change are potentially involved in regulation of target genes related to neuronal dysfunction in response to RABV infection.

\section{Discussion}

As the perturbation of gene regulation networks based on miRNA activities may result in abnormal brain function (Im et al., 2012), it is expected that the highly neurotropic 
Table 2. Significant neuronal function-related GO annotations for the downregulated miRNAs

\begin{tabular}{|c|c|c|c|c|}
\hline GO term & Count $^{\mathrm{a}}$ & $\%^{\mathrm{b}}$ & $P$-value ${ }^{c}$ & $\mathrm{FDR}^{\mathrm{d}}$ \\
\hline \multicolumn{5}{|l|}{ Biological process } \\
\hline GO:0035295 tube development & 159 & 2.890384 & $1.02 \mathrm{E}-25$ & $1.93 \mathrm{E}-22$ \\
\hline GO:0030182 neuron differentiation & 209 & 3.799309 & $9.92 \mathrm{E}-23$ & $1.88 \mathrm{E}-19$ \\
\hline GO:0048666 neuron development & 161 & 2.926741 & $1.49 \mathrm{E}-20$ & $2.82 \mathrm{E}-17$ \\
\hline GO:0031175 neuron projection development & 129 & 2.345028 & $4.28 \mathrm{E}-20$ & $8.10 \mathrm{E}-17$ \\
\hline GO:0048812 neuron projection morphogenesis & 108 & 1.963279 & $1.42 \mathrm{E}-18$ & $2.69 \mathrm{E}-15$ \\
\hline GO:0016192 vesicle-mediated transport & 221 & 4.017451 & 2.63E-17 & $4.97 \mathrm{E}-14$ \\
\hline GO:0007409 axonogenesis & 100 & 1.817851 & $2.96 \mathrm{E}-17$ & $5.59 \mathrm{E}-14$ \\
\hline GO:0007264 small GTPase mediated signal transduction & 137 & 2.490456 & $1.19 \mathrm{E}-15$ & $2.31 \mathrm{E}-12$ \\
\hline GO:0007411 axon guidance & 66 & 1.199782 & $2.49 \mathrm{E}-14$ & $4.70 \mathrm{E}-11$ \\
\hline GO:0030029 actin filament-based process & 95 & 1.726959 & $1.13 \mathrm{E}-11$ & $2.14 \mathrm{E}-08$ \\
\hline GO:0030036 actin cytoskeleton organization & 87 & 1.581531 & 4.37E-10 & $8.26 \mathrm{E}-07$ \\
\hline GO:0030001 metal ion transport & 190 & 3.453917 & $4.46 \mathrm{E}-10$ & $8.44 \mathrm{E}-07$ \\
\hline GO:0030900 forebrain development & 84 & 1.526995 & $1.60 \mathrm{E}-08$ & $3.03 \mathrm{E}-05$ \\
\hline GO:0007423 sensory organ development & 117 & 2.126886 & $3.22 \mathrm{E}-08$ & $6.10 \mathrm{E}-05$ \\
\hline GO:0006811 ion transport & 274 & 4.980913 & 4.19E-08 & 7.92E-05 \\
\hline GO:0035023 regulation of Rho protein signal transduction & 50 & 0.908926 & $6.50 \mathrm{E}-08$ & $1.23 \mathrm{E}-04$ \\
\hline GO:0007268 synaptic transmission & 86 & 1.563352 & $1.09 \mathrm{E}-07$ & $2.06 \mathrm{E}-04$ \\
\hline GO:0007010 cytoskeleton organization & 140 & 2.544992 & $1.12 \mathrm{E}-07$ & $2.12 \mathrm{E}-04$ \\
\hline GO:0043087 regulation of GTPase activity & 51 & 0.927104 & $5.58 \mathrm{E}-07$ & 0.001055 \\
\hline GO:0019226 transmission of nerve impulse & 101 & 1.83603 & $8.82 \mathrm{E}-07$ & 0.001668 \\
\hline GO:0000165 MAPKKK cascade & 58 & 1.054354 & $2.14 \mathrm{E}-06$ & 0.004047 \\
\hline GO:0030902 hindbrain development & 39 & 0.708962 & $2.24 \mathrm{E}-06$ & 0.004235 \\
\hline GO:0001764 neuron migration & 40 & 0.727141 & $3.02 \mathrm{E}-06$ & 0.005709 \\
\hline GO:0050808 synapse organization & 31 & 0.563534 & $3.04 \mathrm{E}-06$ & 0.005744 \\
\hline GO:0006836 neurotransmitter transport & 43 & 0.781676 & $9.74 \mathrm{E}-06$ & 0.018427 \\
\hline GO:0012501 programmed cell death & 180 & 3.272132 & $2.26 \mathrm{E}-05$ & 0.042725 \\
\hline \multicolumn{5}{|l|}{ Cellular component } \\
\hline GO:0045202 synapse & 180 & 3.272132 & $1.36 \mathrm{E}-28$ & $2.03 \mathrm{E}-25$ \\
\hline GO:0043005 neuron projection & 136 & 2.472278 & $6.63 \mathrm{E}-21$ & $9.89 \mathrm{E}-18$ \\
\hline GO:0031982 vesicle & 218 & 3.962916 & $9.88 \mathrm{E}-14$ & $1.48 \mathrm{E}-10$ \\
\hline GO:0005856 cytoskeleton & 403 & 7.325941 & $1.09 \mathrm{E}-11$ & $1.62 \mathrm{E}-08$ \\
\hline GO:0014069 postsynaptic density & 38 & 0.690783 & $1.66 \mathrm{E}-11$ & $2.48 \mathrm{E}-08$ \\
\hline GO:0030425 dendrite & 64 & 1.163425 & $2.22 \mathrm{E}-11$ & $3.31 \mathrm{E}-08$ \\
\hline GO:0019717 synaptosome & 42 & 0.763498 & 2.48E-09 & $3.70 \mathrm{E}-06$ \\
\hline GO:0030424 axon & 58 & 1.054354 & 7.96E-09 & $1.19 \mathrm{E}-05$ \\
\hline GO:0045211 postsynaptic membrane & 63 & 1.145246 & $9.06 \mathrm{E}-08$ & $1.35 \mathrm{E}-04$ \\
\hline GO:0015629 actin cytoskeleton & 91 & 1.654245 & $1.35 \mathrm{E}-07$ & $2.01 \mathrm{E}-04$ \\
\hline GO:0034702 ion channel complex & 71 & 1.290674 & $2.17 \mathrm{E}-06$ & 0.003238 \\
\hline GO:0034703 cation channel complex & 48 & 0.872569 & $2.62 \mathrm{E}-06$ & 0.003913 \\
\hline GO:0030027 lamellipodium & 35 & 0.636248 & $5.57 \mathrm{E}-06$ & 0.008319 \\
\hline GO:0012506 vesicle membrane & 47 & 0.85439 & $1.40 \mathrm{E}-05$ & 0.02084 \\
\hline GO:0043197 dendritic spine & 19 & 0.345392 & $1.98 \mathrm{E}-05$ & 0.029622 \\
\hline GO:0042641 actomyosin & 19 & 0.345392 & $1.98 \mathrm{E}-05$ & 0.029622 \\
\hline GO:0005905 coated pit & 21 & 0.381749 & $2.83 \mathrm{E}-05$ & 0.042257 \\
\hline
\end{tabular}

RABV would also affect neuronal miRNA expression. In previous studies, miRNA expression profiles of mouse brains infected with street or fixed RABV were described. Functional analysis showed that differentially expressed miRNAs were mainly involved in many immune-related signaling pathways, such as the Jak-STAT signaling pathway, cytokine-cytokine receptor interactions, and Fc gamma R-mediated phagocytosis (Zhao et al., 2012a,b). Because neurons are outnumbered by the various nonneuronal supportive cellular elements in the brain, such as glial cells, cells of the blood vessels and cells of the choroid plexus, miRNA expression patterns from whole-brain may be somewhat difficult to unravel miRNA dysregulation in neuron induced by RABV. 
Table 3. Significant neuronal function-related biological pathways

\begin{tabular}{|c|c|c|c|c|}
\hline KEGG Pathway & Count $^{\mathrm{a}}$ & $\%^{\mathrm{b}}$ & $P$-value & $\mathrm{FDR}^{\mathrm{d}}$ \\
\hline \multicolumn{5}{|l|}{ For upregulated miRNAs } \\
\hline mmu04360:Axon guidance & 64 & 1.651613 & $4.56 \mathrm{E}-14$ & $5.62 \mathrm{E}-11$ \\
\hline mmu04310:Wnt signaling pathway & 65 & 1.677419 & $1.70 \mathrm{E}-11$ & $2.10 \mathrm{E}-08$ \\
\hline mmu04810:Regulation of actin cytoskeleton & 80 & 2.064516 & $1.33 \mathrm{E}-09$ & $1.63 \mathrm{E}-06$ \\
\hline mmu04722:Neurotrophin signaling pathway & 52 & 1.341935 & $7.85 \mathrm{E}-08$ & $9.67 \mathrm{E}-05$ \\
\hline mmu04150:mTOR signaling pathway & 26 & 0.670968 & $5.52 \mathrm{E}-06$ & 0.006796 \\
\hline mmu04120:Ubiquitin mediated proteolysis & 48 & 1.23871 & $1.59 \mathrm{E}-05$ & 0.019562 \\
\hline \multicolumn{5}{|l|}{ For downregulated miRNAs } \\
\hline mmu04360:Axon guidance & 86 & 1.563352 & $5.15 \mathrm{E}-20$ & $6.41 \mathrm{E}-17$ \\
\hline mmu04010:MAPK signaling pathway & 139 & 2.526813 & $5.68 \mathrm{E}-19$ & 7.07E-16 \\
\hline mmu04722:Neurotrophin signaling pathway & 76 & 1.381567 & $1.07 \mathrm{E}-13$ & $1.33 \mathrm{E}-10$ \\
\hline mmu04810:Regulation of actin cytoskeleton & 110 & 1.999636 & $1.19 \mathrm{E}-13$ & $1.48 \mathrm{E}-10$ \\
\hline mmu04310:Wnt signaling pathway & 80 & 1.454281 & $9.79 \mathrm{E}-12$ & $1.22 \mathrm{E}-08$ \\
\hline mmu04150:mTOR signaling pathway & 34 & 0.618069 & $1.43 \mathrm{E}-07$ & $1.77 \mathrm{E}-04$ \\
\hline mmu04350:TGF-beta signaling pathway & 47 & 0.85439 & $2.50 \mathrm{E}-07$ & $3.12 \mathrm{E}-04$ \\
\hline mmu04720:Long-term potentiation & 38 & 0.690783 & $3.79 \mathrm{E}-06$ & 0.004715 \\
\hline
\end{tabular}

In order to understand how cellular miRNAs play roles in neuronal dysfunction caused by RABV infection, we performed miRNA microarray assay in RABV-infected primocultures of mouse neurons. In our study, we have demonstrated that a number of miRNAs were significantly differentially expressed during RABV infection in a timedependent manner. Some miRNAs have been well known for their important roles in neuronal development, function and pathology. MiR-9 exerts diverse cell-autonomous effects on the proliferation, migration and differentiation of neural progenitor cells by modulating different mRNA targets (Yuva-Aydemir et al., 2011). Dysregulation of miR-9 in postmitotic neurons is also implicated in brain function and neurodegenerative diseases (Lukiw, 2007; Packer et al., 2008). As one of the most highly conserved neuronalspecific miRNAs, miR-124 is involved in diversity in dendrite morphology, larval locomotion, synaptic release at the neuromuscular junction and neuronal fate determination (Visvanathan et al., 2007; Sun et al., 2012). Another brainspecific miRNA, miR-132, is linked to multiple functions, including neuronal cell development and synaptic plasticity (Lambert et al., 2010; Tognini et al., 2012). Precise control of SNAP-25 by miR-153 is necessary not only for presynaptic vesicle release, but also for protein secretion, motor neuron patterning, and outgrowth (Wei et al., 2013). MiR-181a is a key regulator of mammalian AMPA-type glutamate receptors with potential implications for the regulation of drug-induced synaptic plasticity (Saba et al., 2012). Targeting of Arpc3 by miR-29a/b fine-tunes structural plasticity by regulating actin network branching in mature and developing spines (Lippi et al., 2011).
Previous observations suggest that neuronal dysfunction, rather than neuronal death, is likely responsible for the clinical disease and fatal outcome in rabies under natural conditions, such as electrophysiological alterations, effects on ion channels and neurotransmission (Gourmelon et al., 1991; Iwata et al., 2000; Fu et al., 2005). To better understand the potential role of these differentially expressed miRNAs in RABV-induced neuronal dysfunction, we predicted target genes and performed bioinformatics analysis. Neuronal function-related GO biological processes were overrepresented, such as vesicle-mediated transport, synaptic transmission, axon guidance, synapse organization and neurotransmitter transport. The predicted target genes were also enriched into specific cellular components of neuron, such as synapse, axon, dendrite, synaptosome. Neuronal function depends on proper pathway activity. By pathway enrichment analysis, we found that predicted target genes of differentially expressed miRNAs upon rabies virus infection could be enriched into neuronal function-related pathway, such as axon guidance, neurotrophin signaling pathway, regulation of actin cytoskeleton, mTOR signaling pathway, long-term potentiation. These pathways regulate many aspects of neuron, including neuronal survival, differentiation, development, axon growth and navigation, dendritic arborization, synapse formation and synaptic plasticity (Reichardt, 2006; Swiech et al., 2008; Park et al., 2012). These results suggest that RABV causes an imbalance in host gene expression and intracellular signaling pathway of neuron potentially by modulating host miRNA expression, which may lead to malfunction of neuron or favor viral life cycle. 
In conclusion, our study unveiled the RABV-induced dysregulation of miRNA in primary neurons of mice, whose expression patterns and bioinformatics analysis provide a useful clue for understanding of the neuronal dysfunction caused by RABV infection. Further investigation is needed to clarify the roles of identified miRNA in the pathogenesis of RABV.

Acknowledgements. This work was supported by grants from National Natural Science Foundation of China (grant No. 31172337) and Key Laboratory of Jilin Province for zoonosis prevention and control - Province of State Key Laboratory cultivation base construction the grant (grant No. 2012ZPC).

\section{References}

Ambros V (2004): The functions of animal microRNAs. Nature 431, 350-355. http://dx.doi.org/10.1038/nature02871

Anastasiadou E, Boccellato F, Vincenti S, Rosato P, Bozzoni I, Frati L, Faggioni A, Presutti C, Trivedi P (2010): Epstein-Barr virus encoded LMP1 downregulates TCL1 oncogene through miR-29b. Oncogene 29, 1316-1328. http:// dx.doi.org/10.1038/onc.2009.439

Bartel DP (2004): MicroRNAs: genomics, biogenesis, mechanism, and function. Cell 116, 281-297. http://dx.doi. org/10.1016/S0092-8674(04)00045-5

Bellon M, Lepelletier Y, Hermine O, Nicot C (2009): Deregulation of microRNA involved in hematopoiesis and the immune response in HTLV-I adult T-cell leukemia. Blood 113, 4914-4917. http://dx.doi.org/10.1182/blood-2008-11$\underline{189845}$

Cullen BR (2010): Five questions about viruses and microRNAs. PLoS Pathog. 6, e1000787. http://dx.doi.org/10.1371/ journal.ppat.1000787

Fu ZF, Jackson AC (2005): Neuronal dysfunction and death in rabies virus infection. J. Neurovirol. 11, 101-106. http://dx.doi. org/10.1080/13550280590900445

Gourmelon P, Briet D, Clarencon D, Court L, Tsiang H (1991): Sleep alterations in experimental street rabies virus infection occur in the absence of major EEG abnormalities. Brain Res. 554, 159-165. http://dx.doi.org/10.1016/0006-8993(91) $90184-\mathrm{W}$

Guan Z, Shi N, Song Y, Zhang X, Zhang M, Duan M (2012): Induction of the cellular microRNA-29c by influenza virus contributes to virus-mediated apoptosis through repression of antiapoptotic factors BCL2L2. Biochem. Biophys. Res. Commun. 425, 662-667. http://dx.doi.org/10.1016/j. bbrc.2012.07.114

Hemachudha T, Laothamatas J, Rupprecht CE (2002): Human rabies: a disease of complex neuropathogenetic mechanisms and diagnostic challenges. Lancet Neurol. 1, 101-109. http://dx.doi.org/10.1016/S1474-4422(02)00041-8

Hochberg Y, Benjamini Y (1990): More powerful procedures for multiple significance testing. Stat. Med. 9, 811-818. http:// dx.doi.org/10.1002/sim.4780090710
Huang da W, Sherman BT, Lempicki RA (2009): Bioinformatics enrichment tools: paths toward the comprehensive functional analysis of large gene lists. Nucleic Acids Res. 37, 1-13. http://dx.doi.org/10.1093/nar/gkn923

Im HI, Kenny PJ (2012): MicroRNAs in neuronal function and dysfunction. Trends Neurosci. 35, 325-334. http://dx.doi. org/10.1016/j.tins.2012.01.004

Iwata M, Unno T, Minamoto N, Ohashi H, Komori S (2000): Rabies virus infection prevents the modulation by alpha(2)adrenoceptors, but not muscarinic receptors, of $\mathrm{Ca}(2+)$ channels in NG108-15 cells. Eur. J. Pharmacol. 404, 7988. http://dx.doi.org/10.1016/S0014-2999(00)00621-X

Jogai S, Radotra BD, Banerjee AK (2000): Immunohistochemical study of human rabies. Neuropathology 20, 197-203. http://dx.doi.org/10.1046/j.1440-1789.2000.00332.x

Jopling CL, Yi M, Lancaster AM, Lemon SM, Sarnow P (2005): Modulation of hepatitis $C$ virus RNA abundance by a liver-specific MicroRNA. Science 309, 1577-1581. http://dx.doi.org/10.1126/science.1113329

Lambert TJ, Storm DR, Sullivan JM (2010): MicroRNA132 modulates short-term synaptic plasticity but not basal release probability in hippocampal neurons. PLoS One 5, e15182. http://dx.doi.org/10.1371/journal.pone.0015182

Lewis BP, Burge CB, Bartel DP (2005): Conserved seed pairing, often flanked by adenosines, indicates that thousands of human genes are microRNA targets. Cell 120, 15-20. http://dx.doi.org/10.1016/j.cell.2004.12.035

Lippi G, Steinert JR, Marczylo EL, D'Oro S, Fiore R, Forsythe ID, Schratt G, Zoli M, Nicotera P, Young KW (2011): Targeting of the Arpc3 actin nucleation factor by miR-29a/b regulates dendritic spine morphology. J. Cell Biol. 194, 889-904. http://dx.doi.org/10.1083/jcb.201103006

Lukiw WJ (2007): Micro-RNA speciation in fetal, adult and Alzheimer's disease hippocampus. Neuroreport 18, 297-300. http://dx.doi.org/10.1097/WNR.0b013e3280148e8b

Packer AN, Xing Y, Harper SQ, Jones L, Davidson BL (2008): The bifunctional microRNA miR-9/miR-9* regulates REST and CoREST and is downregulated in Huntington's disease. J. Neurosci. 28, 14341-14346. http://dx.doi.org/10.1523/ INEUROSCI.2390-08.2008

Park M, Shen K (2012): WNTs in synapse formation and neuronal circuitry. EMBO J. 31, 2697-2704. http://dx.doi. org/10.1038/emboj.2012.145

Reichardt LF (2006): Neurotrophin-regulated signalling pathways. Philos. Trans. R. Soc. Lond. B Biol. Sci. 361, 1545-1564. http://dx.doi.org/10.1098/rstb.2006.1894

Rom S, Rom I, Passiatore G, Pacifici M, Radhakrishnan S, Del Valle L, Pina-Oviedo S, Khalili K, Eletto D, Peruzzi F (2010): CCL8/MCP-2 is a target for mir-146a in HIV-1-infected human microglial cells. FASEB J. 24, 2292-2300. http:// dx.doi.org/10.1096/fi.09-143503

Saba R, Storchel PH, Aksoy-Aksel A, Kepura F, Lippi G, Plant TD, Schratt, GM (2012): Dopamine-regulated microRNA MiR-181a controls GluA2 surface expression in hippocampal neurons. Mol. Cell Biol. 32, 619-632. http://dx.doi. org/10.1128/MCB.05896-11 
Schnell MJ, McGettigan JP, Wirblich C, Papaneri A (2010): The cell biology of rabies virus: using stealth to reach the brain. Nat. Rev. Microbiol. 8, 51-61.

Sun K, Westholm JO, Tsurudome K, Hagen JW, Lu Y, Kohwi M, Betel D, Gao FB, Haghighi AP, Doe CQ, Lai EC (2012): Neurophysiological defects and neuronal gene deregulation in Drosophila mir-124 mutants. PLoS Genet. 8, e1002515. http://dx.doi.org/10.1371/journal.pgen.1002515

Swiech L, Perycz M, Malik A, Jaworski J (2008): Role of mTOR in physiology and pathology of the nervous system. Biochim. Biophys. Acta 1784, 116-132. http://dx.doi. org/10.1016/j.bbapap.2007.08.015

Tognini P, Pizzorusso T (2012): MicroRNA212/132 family: molecular transducer of neuronal function and plasticity. Int. J. Biochem. Cell Biol. 44, 6-10. http://dx.doi.org/10.1016/j. biocel.2011.10.015

Visvanathan J, Lee S, Lee B, Lee JW, Lee SK (2007): The microRNA miR-124 antagonizes the anti-neural REST/ SCP1 pathway during embryonic CNS development. Genes Dev. 21, 744-749. http://dx.doi.org/10.1101/ gad. 1519107

Wang FZ, Weber F, Croce C, Liu CG, Liao X, Pellett PE (2008): Human cytomegalovirus infection alters the expression of cellular microRNA species that affect its replication. J. Virol. 82, 9065-9074. http://dx.doi.org/10.1128/ JVI.00961-08

Wei C, Thatcher EJ, Olena AF, Cha DJ, Perdigoto AL, Marshall AF, Carter BD, Broadie K, Patton JG (2013): miR-153 regulates SNAP-25, synaptic transmission, and neuronal development. PLoS One 8, e57080. http://dx.doi. org/10.1371/journal.pone.0057080

Yuva-Aydemir Y, Simkin A, Gascon E, Gao FB (2011): MicroRNA-9: functional evolution of a conserved small regulatory RNA. RNA Biol. 8, 557-564. http://dx.doi. org/10.4161/rna.8.4.16019

Zhao P, Zhao L, Zhang K, Feng H, Wang H, Wang T, Xu T, Feng N, Wang C, Gao Y, Huang G, Qin C, Yang S, Xia X (2012a): Infection with street strain rabies virus induces modulation of the microRNA profile of the mouse brain. Virol. J. 9, 159. http://dx.doi.org/10.1186/1743-422X-9-159

Zhao P, Zhao L, Zhang T, Wang H, Qin C, Yang S, Xia X (2012b): Changes in microRNA expression induced by rabies virus infection in mouse brains. Microb. Pathog. 52, 47-54. http://dx.doi.org/10.1016/j.micpath.2011.10.001 